\title{
II ruolo della Cardiologia Riabilitativa nei programmi di disease management dello scompenso cardiaco
}

\author{
Stefano Urbinati
}

Monaldi Arch Chest Dis 2006; 66: 139-141.

UOC Cardiologia, Ospedale Bellaria, Bologna.

La gestione dello scompenso cardiaco, così come è organizzata oggi, risulta ancora inadeguata per le sfide che questa patologia ci propone, malgrado i progressi terapeutici e organizzativi compiuti. Dopo un ricovero per scompenso cardiaco infatti l'incidenza di riospedalizzazioni è del $50-60 \%$ a sei mesi e queste sono frequenti soprattutto nelle prime 2-4 settimane dall'evento acuto [1]. Alla luce di questi risultati probabilmente non è più scontato che una percentuale così irrisoria di pazienti (circa il 20\%) debba essere gestita in Cardiologia nella fase di instabilizzazione, non è più scontato che il paziente debba essere dimesso senza informazioni sullo stile di vita da tenere, e senza una pianificazione del follow-up che dovrà seguire e di come autogestire alcune terapie.

La situazione attuale è evidentemente insoddisfacente e necessita di proposte operative originali per essere affrontata. In particolare sono argomento di discussione: 1) le sedi dove ricoverare i pazienti con scompenso cardiaco; 2) le modalità di pianificazione del follow-up; 3) come sviluppare un network locale che preveda percorsi differenziati per tipologie diverse di pazienti, oppure per lo stesso paziente nelle diverse fasi della sua storia naturale poiché un percorso unico non è ipotizzabile data l'eterogeneità dei pazienti con scompenso cardiaco. Per effettuare una risposta efficace è necessario tener conto della rete cardiologica locale, la cui definizione è stata fortemente auspicata nel documento ANMCO-SIC del 2003 [2] e anche dal recente documento di consenso promosso dall'Area Scompenso dell'ANMCO [3]. Anche le più recenti linee guida internazionali suggeriscono che solo con una adeguata organizzazione dell'assistenza si può migliorare la qualità della vita e ridurre le riospedalizzazioni [4]. Si tratta di una raccomandazione di Classe 1, livello di evidenza A. Per realizzare una rete locale però bisogna tener conto della disponibilità di risorse e dell'organizzazione pre-esistente molto importante in un periodo di devolution sanitaria in cui le realtà sanitarie locali sono molto diverse l'una dall'altra.

La prima cosa che a mio parere va ridefinita è la sede dove ricoverare i pazienti. La situazione attuale $(80 \%$ dei pazienti in reparti internistici, il $20 \%$ in cardiologia) è insoddisfacente. Si possono ipotizzare tre possibili alternative 1) dimensionare le UTIC in modo da renderle disponibili a ricoverare una percentuale maggiore di pazienti con scompenso car- diaco. Personalmente non so se questo sia effettivamente possibile, anche perché in questa fase la definizione delle reti per le sindromi coronariche acute sta monopolizzando la maggior parte delle risorse e non rende praticabile questo percorso anche se, naturalmente, è un elemento da considerare nella propria realtà locale. 2) realizzare reparti " $a d$ hoc" per lo scompenso cardiaco, eventualmente a gestione mista cardiologica e internistica. Su questo tema ci sono esperienze molto interessanti a livello internazionale ed anche a livello italiano [5]: dove esistono, possono essere utilizzate in tal senso anche le Unità Operative di Cardiologia Riabilitativa che finora, secondo i dati del censimento più recente, hanno investito relativamente poco sullo scompenso cardiaco [6]. È possibile che alcune Cardiologie Riabilitative " $h u b$ " possano svolgere un ruolo in questo senso se dispongono di una terapia sub-intensiva dove sia possibile l'utilizzo di sofisticate tecniche di diagnostica non invasiva e la possibilità di eseguire un cateterismo destro, un approccio sistematico alla terapia farmacologica e non farmacologica, una valutazione ergometrica occupazionale e la gestione di comorbidità e situazioni complesse come suggerito anche dalle recenti linee guida dell'Agenzia Sanitaria per i Servizi Regionali [7]. Ma chi sono i pazienti con problematiche complesse? Sono quelli affetti da scompenso cardiaco che necessitano di terapie infusive, pazienti ad alto rischio di instabilizzazione, quelli sopravvissuti ad arresto cardiaco. Oppure anche pazienti che presentano una serie di comorbidità, come quelli in genere accolti in reparti internistici, che probabilmente in reparti cardiologici governati da team multidisciplinari potrebbero ricevere un trattamento più aggressivo e forse più efficace, in termini di risultati, sul follow-up. Non è attualmente disponibile una evidenza scientifica su questo tipo di ipotesi, perché non sono stati eseguiti grandi trials sull'argomento.

Altro elemento fondamentale da affrontare è la pianificazione del follow-up: infatti da qualsiasi reparto un paziente con scompenso cardiaco venga dimesso è assolutamente necessario e improcrastinabile che venga pianificato il percorso post-dimissione. Negli ultimi 10 anni sono stati pubblicati molti studi sull'efficacia di programmi di disease management sullo scompenso cardiaco, una recente metanalisi ha concluso che la pianificazione del followup alla dimissione è assolutamente cost-effective, 
perché riduce le riospedalizzazioni del $25 \%$, con un abbattimento importante dei costi e anche della mortalità (8-10\%) [8], anche se purtroppo questo rapporto di continuità tra ospedale e territorio non costituisce la norma [9]. Alla dimissione dunque è necessario prevedere percorsi prestabiliti. Quali percorsi sono proponibili? Quello che fa riferimento all'ambulatorio cardiologico dedicato ma anche quello per il paziente internistico-geriatrico, per cui è preferibile un programma di disease management territoriale con un forte supporto educazionale e che si avvalga di infermieri dedicati. La realizzazione di questi programmi di disease management però deve tener conto della realtà eterogenea che si trova sul territorio, perché è complesso interagire con centinaia di medici di famiglia e il rapporto tra infermieri e medici di medicina generale non è standardizzato come avviene in ospedale.

Negli ultimi anni accanto all'abbondante letteratura copiosa sui programmi di disease management stanno emergendo dati sull'efficacia di sistemi di telemedicina o di monitoraggio poliparametrico attraverso reti informatiche. Per esempio Cleland et al. [10] in uno studio recente hanno dimostrato che un telemonitoraggio può dare lo stesso risultato ottenuto con il supporto infermieristico.

Un tema a parte è quello relativo al ruolo che può svolgere la Cardiologia Riabilitativa nella gestione di pazienti con scompenso cardiaco avanzato e refrattario. La fase che segue una instabilizzazione rappresenta un terreno "minato" perché sappiamo che la maggior parte delle riospedalizzazioni si verifica nelle prime 3-4 settimane dopo l'evento acuto. In questa fase è necessario porsi degli obiettivi chiari:

1. stratificare il rischio per stabilire l'intensità del monitoraggio successivo,

2. verificare la stabilità clinica correggendo i fattori precipitanti l'instabilità, problema spesso sottostimato,

3. identificare il fattore precipitante che ha determinato l'instabilità clinica,

4. raggiungere goal terapeutici, che significa modificare il dosaggio dei farmaci con una cadenza a volte addirittura quotidiana.

Inoltre, in un settore come quello riabilitativo, è possibile attuare in maniera strutturata un programma di training fisico ed educazionale (che si può iniziare a stabilizzazione avvenuta) ed interventi specifici per le comorbidità, come ad esempio il percorso diabete. Negli studi presi in considerazione dalla metanalisi sui programmi di disease management non erano previsti cicli di training fisico e questa mi sembra una carenza molto importante in considerazione del livello di evidenza di cui dispone oggi il training fisico sul miglioramento della qualità della vita e sulla capacità funzionale dei pazienti. Per esempio tra i soggetti che hanno una frazione d'eiezione del $20 \%$, possiamo trovare pazienti con capacità funzionale molto depressa $\left(\mathrm{VO}_{2} \max \cong 10\right.$ $\mathrm{ml} / \mathrm{Kg} / \mathrm{m}$ ) e altri con una buona tolleranza allo sforzo che hanno una vita quasi normale $\left(\mathrm{VO}_{2} \max \cong 20\right.$ $\mathrm{ml} / \mathrm{Kg} / \mathrm{m}$ ) e sappiamo che con un ciclo di training fisico possiamo migliorare il $\mathrm{VO}_{2}$ max mediamente del 20\% [11]. Naturalmente il training fisico deve essere trattato come qualsiasi altra terapia e quindi deve essere somministrato secondo modalità, dura- ta, intensità, frequenza codificate. Le linee guida pubblicate negli ultimi anni ci danno ragguagli molto precisi su come deve essere condotto e sul fatto che, solo un paziente stabile da almeno 3 settimane, possa accedere ad un ciclo di training fisico in maniera sicura [12].

L'intervento riabilitativo è in grado di migliorare la sopravvivenza come dimostrato in uno studio della Duke University [13] ed anche in quello di Montescano [14], che ha dimostrato che con un day hospital riabilitativo è possibile ottenere lo stesso risultato sulla sopravvivenza con una interessante riduzione dei costi. Uno studio inglese pubblicato nel maggio 2005 [15] ha mostrato l'efficacia di un programma di disease management in cui viene inserito, accanto al programma educazionale, anche un programma di training fisico di 16 settimane. In questo studio, riferito a pazienti anziani, si è osservato non solo un miglioramento di tutti i parametri, ma anche una riduzione delle ospedalizzazioni. Altri sono studi in corso come quello danese, che forse è lo studio quantitativamente più importante, presentato lo scorso novembre sull'American Heart Journal [16] e tuttora in corso.

In conclusione, le sfide proposte dall'esplosione epidemiologica dello scompenso cardiaco richiedono soluzioni organizzative innovative ed originali che devono essere ben integrate con l'assistenza disponibile nel proprio territorio di riferimento. Innanzitutto è necessario riconsiderare quale sia la sede più idonea dove ricoverare un paziente con scompenso cardiaco nella fase acuta, considerare la necessità di definire follow-up programmati alla dimissione e, in ultima analisi, realizzare un network locale che preveda opzioni diverse per pazienti con problemi diversi.

Recentemente nella nostra realtà di Bologna si sta sviluppando un particolare tipo di network che prevede che pazienti dimessi dalle medicine interne e dalle geriatrie seguano prevalentemente un programma di disease management a livello territoriale, mentre quelli dimessi dalle cardiologie, prima di accedere al follow-up gestito dall'ambulatorio dedicato, sono ammessi preferibilmente per un ciclo di day hospital di 4-6 settimane di riabilitazione cardiologica. Questo evidenzia come la Cardiologia Riabilitativa possa a pieno diritto inserirsi nella "rete" per la gestione del follow-up nel paziente con scompenso cardiaco.

\section{Bibliografia}

1. Di Lenarda A, Scherillo M, Maggioni AP, et al for the Temistocle Investigators. Current presentation and management of heart failure in cardiology and internal medicine hospital units: a tale of two worlds: the TEMISTOCLE study. Am Heart J 2003; 146: E12.

2. Federazione Italiana di Cardiologia. Struttura e Organizzazione funzionale della Cardiologia. Ital Heart J suppl 2003; 4 881-930.

3. Consensus Conference: Il percorso assistenziale del paziente con scompenso cardiaco. G Ital Cardiol 2006; 7 : 387-432.

4. Hunt SA, Abraham WT, Chin MH, et al. ACC/AHA 2005 guideline update for the diagnosis and management of chronic heart failure in the adult. A Report of the ACC/AHA Task Force on Practice Guidelines. J Am Coll Cardiol 2005; 46: 1116-43. 
5. Senni M, Gori M, Alari G, et al. Unità di Medicina Cardiovascolare: prima esperienza di un approccio intraospedaliero multidisciplinare allo scompenso cardiaco. G Ital Cardiol 2006; 1: 348-358.

6. Urbinati S, Fattirolli F, Tramarin R, et al. The ISYDE project. A survey on cardiac rehabilitation in Italy. Monaldi Arch Chest Dis 2003; 60: 16-24.

7. Agenzia Sanitaria per i Servizi Regionali (ASSR). Linee guida nazionali sulla Riabilitazione Cardiologica, 2006, www.assr.it e Monaldi Arch Chest Dis (in press).

8. Gonseth, et al. The effectiveness of disease management programmes reducing hospital readmissions in older patients with heart failure: a systematic review and metanalysis of published reports. Eur Heart $J$ 2004; 25: 1570-95.

9. Rumsfeld JS, Masoudi FA. Heart failure disease management works, but will it succeeded? Eur Heart J 2004; 25 : 1593-1604.

10. Cleland JGF, Luois AA, Rigby AS, Janssens U, Balk AHM, for the TEN-HMS Investigators. Noninvasive home telemonitoring for patients at high risk of recurrent admission and death. J Am Coll Cardiol 2005; 45: 16541664.

11. Urbinati S, Pergolini F, Marchetti G, Pinelli G. Interazione tra training fisico, terapia farmacologica e rimodella- mento ventricolare nel paziente con disfunzione ventricolare sinistra. Ital Heart J 2000; 1: 1545-1552.

12. Working Group on Cardiac Rehabilitation and Exercise Physiology and Working Group on Heart Failure of European Society of Cardiology. Recommendations for exercise testing in chronic heart failure. Eur Heart $J$ 2001; 22: 37-45.

13. Whellan DJ, Shaw LK, Bart BA, et al. Cardiac rehabilitation and survival in patients with left ventricular systolic dysfunction. Am Heart $J$ 2001; 142: 160-6.

14. Capomolla S, Febo O, Ceresa M, et al. Cost-utility ratio in chronic heart failure: comparison between heart failure management program delivered by day-hospital and usual care. J Am Coll Cardiol 2002; 40: 1259-66.

15. Austin J, Williams R, Ross L, et al. Randomised controlled trial of cardiac rehabilitation in elderly patients with heart failure. Eur J Heart Fail 2005; 7: 411-7.

16. Olsen Zwisler AD, Schou L, Boas Soja AM, et al for the DANREHAB Group. A randomized clinical trial of hospital-based comprehensive cardiac rehabilitation versus usual care for patients with congestive heart failure, ischemic heart disease, or high risk of ischemic heart disease (the DANREHAB trial): design, intervention, and population. Am Heart J 2005; 150: 899-e 7-16. 